\title{
Dose dependent impact of rose-hip powder in patients suffering from osteoarthritis of the hip and or knee - a double blind, randomized, placebo controlled, parallel group, phase iii study
}

\begin{abstract}
Objectives: To test if low dose Rose-hip treatment based on seeds and shells, defined as an initial 3 weeks dose of $5 \mathrm{~g} /$ day followed by $2.5 \mathrm{~g} /$ day for another 9 weeks would alleviate symptoms from osteoarthritis.

Methods: 120 patients with osteoarthritis of the hip and/or knee were included in a 12 week placebo controlled, randomized, parallel clinical trial, Clinical Trial. Gov Identifier NCT01459939). Pain, and ADL was estimated using the WOMAC questionnaire.

Results: Primary effect variable, WOMAC pain declined from $17.97+/-8.80$ to $16.57+/$ 9.88 after 12 weeks $(\mathrm{p}<0.141)$ as compared to placebo $17.96+/-7.08$ declining to $14.41+/$ 7.60 after twelve weeks $(\mathrm{p}<0.000), \mathrm{p}<0.058$ comparing groups. WOMAC function (ADL) declined in both groups as the result of 12 weeks treatment: active treatment $60.59+/-31.33$ vs $53.91+/-30.59(\mathrm{p}<0.016)$ and placebo $55.71+/-25.19$ vs $43.90+/-25.43(\mathrm{p}<0.000) \mathrm{p}<$ 0.070 comparing groups. When symptom scores were plotted against the weight of patients in the actively treated group, there was a significant positive correlation between weight of the patient and symptom score, irrespective of the symptom chosen $(\mathrm{p}<0.019$ and 0.009 , respectively). The lower the weight of the patient the greater reduction in pain and ADL symptom scores. In the group treated with placebo there was no correlation between scores and patient weight in any parameter tested $(\mathrm{p}<0813$ and $\mathrm{p}<0.432$, respectively).
\end{abstract}

Conclusions: The present data show that low dose Rose-hip treatment does not reduce symptoms of osteoarthritis when evaluating the entire group of patients. However, lighter patients, clearly benefit from treatment.

Keywords: osteoarthritis, rose-hip, pain, stiffness, womac function, dose-dependence
Volume 7 Issue I - 2017

\author{
Marstrand K,' Warholm L,' ${ }^{2}$ Pedersen F,' Kaj \\ Winther ${ }^{4}$ \\ 'Department of Orthopaedic Surgery, Elverum Hospital, \\ Norway \\ ${ }^{2}$ Department of Trauma Medicine, Horsens Hospital, Denmark \\ ${ }^{3}$ Department of Gynecology, Skejby Hospital, University of \\ Aarhus, Denmark \\ 4Department of Nutrition, Exercise and Sports, University of \\ Copenhagen, Denmark
}

Correspondence: Marstrand K, Department of Orthopaedic Surgery, Elverum Hospital, Kirkeveien 3I, 2409 Elverum, Norway, Tel +47405 I3536, Email kaha@nexs.ku.dk

Received: February 17, 2017| Published: May 08, 2017
Abbreviations: ADL, activity of daily living; CRP, c reactive peptide; ITT, intention to treat; NSAID, non-steroidal antiinflammatory drugs; OA, osteoarthritis; PGAD, patients' global evaluation of disease severity

\section{Introduction}

Osteoarthritis is a common disease among middle aged and elderly people. NSAID`s, paracetamol, codeine and synthetic opioids are well known first choice treatment of symptoms of the disease. Most pain killers, however, have serious side effects such as gastrointestinal bleeding, stomach and gut erosion and kidney damage. ${ }^{1-6}$ Therefore, new treatments, without side effects, which are also chondroprotective, are mandatory. It is also advantageous if such treatments are anti-inflammatory. Recently glucosamine and chondroitin were thought to restore cartilage and to reduce pain. Lately it has been documented that glucosamine and chondroitin are not the complete solution in osteoarthritis. ${ }^{7}$

Recent research including a meta-analysis has shown that current treatment with Rose-hip, in a version containing seeds and shells, is 3times more effective than paracetamol in ameliorating pain. ${ }^{8,9}$ It has been shown that this powder and also an active ingredient, a galactolipid named GOPO isolated from the powder ${ }^{10}$ can confer cartilage protection, ${ }^{11}$ and work as an anti-inflammatory agent. ${ }^{12}$

Up to now the dose of the product has invariably been $5 \mathrm{~g}$ daily. ${ }^{8,9,12}$ Patients have claimed that they felt reduction of symptoms also by taking half the dose. This study was undertaken to test if a lower dosage would alleviate symptoms of osteoarthritis. Specifically, the aim of this study was to test if symptom reduction could be detected after an initial dose of $5 \mathrm{~g}$ Rose-hip daily for 3 weeks, followed by $2.5 \mathrm{~g}$ daily for a following 9 weeks period. A part of the study was also to test the hypotheses: is there any "dose-dependency" in the actively treated group and/or in the placebo group, when defined by a simple correlation analysis testing the weight of patients versus the reported symptom scores. This very simple methodology was earlier applied on a similar group of osteoarthritis patients who were treated with the same herbal remedy as tested in this study, however, at that time in a higher dose. ${ }^{13}$

\section{Study methods and patient sample}

The study included three centres: Aarhus, Horsens and Vejle in eastern Jutland, Denmark. Patients were recruited from announcements in local newspapers. The protocol was approved by the Ethical Committee (No M-20110185), and the Data Supervision (J. nr. 2011-41-6721). Clinical Trial Gov. (Identifier: NCT01459939). Inclusion criteria: age $40+$, males or females with mild to moderate $\mathrm{OA}$ of the hip and/or knee. The risk of a type 1 error was calculated to be less than $5 \%$ and the risk of a type 2 was less than $10 \%{ }^{8,12}$ Osteoarthritis was diagnosed according to the criteria of the American College of Rheumatology. ${ }^{14,15}$ Patients who within the last 3months before the screening were treated with Rose-hip, avocado-soybean, ginger, glucosamine, chondroitin sulphate, TNF-alpha inhibitors or DMARD and patients periodically taking rescue medication like NSAID`s, paracetamol, codeine, and tramadol, were excluded. 
Rescue medication at constant dose throughout the whole study period was accepted. Patients suffering from joint diseases other than OA, abusers of alcohol and drugs, psychiatric diseases, known allergies to rosehip, planned for major surgery, who had participated in another clinical trial within the last 3month and patients who might have changed their eating habits or level of physical activity or having difficulties in collaboration were also excluded.

\section{Design and treatments}

The study was an investigator initiated multicentre, randomized, double blind, placebo-controlled, phase III parallel trial comparing standardized Rose-hip powder to placebo in patients with mild to moderate OA of the knee and/or hip. The duration of the study was 12 weeks. After an initial period of 3 weeks where the dosing was $5 \mathrm{~g}$ Rose-hip daily ( $5+5$ capsules) or placebo the dose was reduced to $2.5 \mathrm{~g}$ daily ( 5 capsules) for the following 9 weeks. Allocation was carried out in blocks of 20 by a computer program. The present Rose-hip powder is produced by Hyben-Vital, Langeland, Denmark (patented methodology) from whole fruits of selected subtypes of Rosa Canina containing shells and seeds. ${ }^{8}$ All capsules were produced from the same batch. Identical capsules containing an inactive powder of similar taste, smell and colour were produced for placebo. Compliance with study treatment was established by counting the number of capsules returned by the patients at each control.

\section{Outcome measures}

Primary endpoints: 1) The impact on WOMAC pain and WOMAC function score after 12 weeks treatment with either Rose-hip or placebo 2) test for a possible "dose-dependency" by plotting patient weight vs. symptom scores.

Secondary endpoints: 1) The Impact on WOMAC pain and function scores after an initial 6weeks of active or placebo treatment 2) The Impact on WOMAC stiffness after 6 and 12 weeks in active and placebo treated patients. 3) The impact of active and placebo treatment on PGAD after 6 and 12weeks treatment. 4) The impact of active treatment and placebo on quality of life using SF-12 questionnaires after 6 and 12weeks treatment. 5) Blood lipid levels and the inflammatory marker CRP using normal laboratory routine. 6) The number of side effects reported in each group.

\section{Statistical measures}

The study was based on ITT, with last value carried forward. Statistical analyses within groups were based on Wilcoxon and comparing groups on Man-Whitney. When focusing on response/no response on the yes/no basis Fisher's test was applied. Correlation analysis was like wise applied. $\mathrm{P} \leq 0.05$ was regarded as statistically significant.

\section{Results}

A total of 202 patients were screened, and 120 randomised. Reasons for exclusion: patients were already using rose-hip, glucosamine or ginger, lack of x-ray verification, osteoarthritis in other joints than hip and/or knee or rheumatoid arthritis.

\section{Demography}

The demography of the two groups is listed in Table 1. Neither group was significantly different regarding smoking habits, coffee intake, physical activity, nor consumption of rescue or prescription medicine observed comparing groups (data not given).

\section{Primary effect variables}

Pain: After 12weeks treatment the group receiving active treatment showed a decline in pain score from $17.97+/-8.80$ to $16.57+/-9.88$ $(\mathrm{p}<0.14)$, whilst the placebo group showed a decline in pain score to $14.41+/-7.60(p<0.001)$. After 12 weeks of treatment there was no statistical difference between the two groups (Table 2).

When the numbers of positive responders (patients reporting a decline in pain after 12 weeks treatment) were counted in each group $53 \%$ in the actively treated group reported a decline in pain and $65 \%$ in the placebo group. No significant difference comparing groups $(\mathrm{p}<0.260)$.

More patients in the actively treated group reported higher pain scores after 6 weeks of treatment than what was observed for placebo. The initial mean pain score in the actively treated group increased insignificantly by $5.5 \%$ (from $17.97+/-8.80$ to $19.03+/-9.94$ ) after 6 weeks $(\mathrm{p}<0.245)$ and its clinical relevance is unclear. Though small, the increase was significant when compared to the placebo group $(\mathrm{p}<0.008)$, Table 2 .

When 6weeks active treatment was compared to that of 12 scores declined from: $19.03+/-9.94$ to $16.57+/-9.88$, (Table 2) the delta decline of $2.47+/-6.07$ was highly significant $(p<0.0005)$. The corresponding delta decline from 6 to 12 weeks placebo was $0.80+/$ 4.92, not statistically significant $(\mathrm{p}<0.118)$.

The drop in pain score from 6 to 12 weeks showed a trend in favour of active treatment, but the Man-Whitney $\mathrm{p}$ level failed to reach significance $(p<0.062)$. It was concluded that treatments did not significantly differ from each other (Table 2 ).

Examination for dose-dependency: The weight of each patient was plotted against the corresponding WOMAC pain symptom score for both active and placebo groups.

Initially no correlation was found between weight and pain scores in either the actively or in the placebo treated group. At 6 and 12 weeks active treatment, however, there was a statistically significant positive correlation between weight and pain score $(\mathrm{p}<0.019$ and $\mathrm{p}<0.019$, respectively). The lower the weight, the more pronounced the reduction in pain (Table 3 ). As evidenced by a $17 \%$ reduction in pain scores in patients with the lowest weight ( 55 to $84 \mathrm{~kg}$ ), whereas patients with weight from 85 to $120 \mathrm{~kg}$ demonstrated a slight increase in pain score of $4.2 \%$. In contrast to the treatment group there was no correlation between weight and pain score at either 6 or $12 \mathrm{weeks}$, in the placebo group $(\mathrm{p}<0.584$ and $\mathrm{p}<0.813)$ (Table 3$)$.

WOMAC function (Activity of Daily Living): Active treatment for 12 weeks resulted in a significant decline (improvement) in ADL score, from $60.59+/-31.33$ to $53.18+/-30.59(\mathrm{p}<0.016)$. There was likewise a decline in the placebo group, from $55.71+/-25.19$ to 43.90 $+/-25.43(\mathrm{p}<0.001)$. No significant difference comparing groups $(p<0.098)$ (Table 2). The percentage of positive responders (patients who reported improved physical activity) in the actively treated group was $58 \%$ vs $64 \%$ in placebo $(\mathrm{p}<0.340)$.

Dose-dependency: Irrespective of treatment, there was no correlation between weight and WOMAC function score initially, (Table 3). Six and 12 weeks of active treatment, however, resulted in a statistically significant positive correlation between weight and WOMAC function score $(p<0.019$ and $p<0.009$, respectively). Again, the lower the weight the more improvement in scores. In the placebo group there was no correlation between weight and WOMAC function score at either time point (Table 3). 
Table I Demography of patients in the active treatment and placebo

\begin{tabular}{llll}
\hline & Active treatment & Placebo treatment & P-Level \\
\hline Women & 34 & 37 & 0.5773 \\
Men & 26 & 23 & 0.2701 \\
Age (Years) & $62.7+/-8.9$ & $64.5+/-10.3$ & 0.418 \\
No.Years with OA & $9.6+/-7.9$ & $9.0+1-8.4$ & 0.87 \\
MBI $\left(\mathrm{kg} / \mathrm{m}^{2}\right)$ & $26.7+/-4.9$ & $26.9+/-5.0$ & 0.7932 \\
Weight $(\mathrm{kg})$ & $79.1+/-16.5$ & $79.7+/-16.5$ & \\
Number of patients with i OA of the: & & & \\
Knee & 36 & 34 & 0.8054 \\
Hip & 12 & 11 & \\
Knee/Hip & 12 & 15 & \\
WOMAC & & & 0.6163 \\
Pain & $17.97+/-8.8$ & $17.96+/-7.1$ & $0.462 I$ \\
Stiffness & $10.48+/-4.3$ & $9.92+/-4.5$ & 0.3926 \\
ADL & $60.59+/-31.3$ & $55.71+/-25.2$ & \\
\hline
\end{tabular}

Table 2 Clinical and biochemical variables of individuals with overweight-obesity

\begin{tabular}{|c|c|c|c|c|c|c|c|c|c|c|}
\hline \multirow[b]{2}{*}{ Item } & \multirow[b]{2}{*}{ Week } & \multicolumn{4}{|c|}{ Active } & \multicolumn{4}{|c|}{ Placebo } & \multirow[b]{2}{*}{ Mann-Whitney } \\
\hline & & $\mathbf{N}$ & Mean & SD & Wilcoxon & $\mathbf{N}$ & Mean & SD & Wilcoxon & \\
\hline \multirow[t]{6}{*}{ Pain } & Week 0 & 60 & 17.97 & 8.80 & & 60 & 17.96 & 7.08 & & 0.6162 \\
\hline & Week 6 & 60 & 19.03 & 9.94 & & 60 & 15.22 & 9.13 & & 0.0271 \\
\hline & Week 12 & 60 & 16.57 & 9.88 & & 60 & $|4.4|$ & 7.60 & & 0.3098 \\
\hline & DIFF 0/6 & 60 & -1.06 & 7.12 & 0.2426 & 60 & 2.74 & 7.51 & 0.0154 & 0.0075 \\
\hline & DIFF $6 / 12$ & 60 & 2.47 & 6.07 & 0.0005 & 60 & 0.80 & 4.92 & 0.1184 & 0.0618 \\
\hline & DIFF 0/I2 & 60 & 1.41 & 7.7I & 0.1413 & 60 & 3.55 & 6.98 & 0.0001 & 0.0580 \\
\hline \multirow[t]{6}{*}{ ADL } & Week 0 & 59 & 60.59 & 31.33 & & 57 & 55.71 & 25.19 & & 0.3920 \\
\hline & Week 6 & 59 & 56.28 & 30.94 & & 57 & 47.69 & 27.81 & & 0.1174 \\
\hline & Week 12 & 59 & 53.91 & 30.59 & & 57 & 43.90 & 25.43 & & 0.0709 \\
\hline & DIFF 0/6 & 59 & 4.32 & 18.96 & 0.0496 & 57 & 8.02 & 20.43 & 0.0019 & 0.1616 \\
\hline & DIFF $6 / 12$ & 59 & 2.37 & 13.99 & 0.1990 & 57 & 3.79 & 13.13 & 0.0116 & 0.5002 \\
\hline & DIFF 0/I2 & 59 & 6.68 & 21.19 & 0.0164 & 57 & |I.8I & 20.89 & 0.0001 & 0.0980 \\
\hline
\end{tabular}

Table 3 Correlation coefficients for weight versus total WOMAC and,WOMAC scores for ADL, Stiffness and Pain.Values are given at the initial level of the trial and after 6 and 12 weeks of treatment. $P$ values are given in brackets $(\# p<0.050)$

\begin{tabular}{lllllll}
\hline & START & & 6 WEEKS & & I W WEEKS \\
\cline { 2 - 6 } Active & Correlation Coefficient & P Value & Correlation Coefficient & P Value & Correlation Coefficient & P Value \\
\hline TotalWOMAC & 0.126 & 0.342 & 0.289 & 0.026 & 0.313 \\
ADL & 0.115 & 0.384 & 0.305 & 0.019 & 0.339 \\
Stiffness & 0.210 & 0.108 & 0.292 & 0.023 & 0.299 \\
Pain & 0.167 & 0.203 & 0.302 & 0.019 & 0.302 & 0.009 \\
Placebo & & & & & \\
TotalWOMAC & 0.075 & 0.592 & 0.068 & 0.606 & 0.093 \\
ADL & 0.089 & 0.513 & 0.053 & 0.694 & 0.106 \\
Stiffness & -0.049 & 0.713 & -0.022 & 0.866 & -0.029 \\
Pain & 0.043 & 0.742 & 0.072 & 0.584 & 0.031 \\
\hline
\end{tabular}

Table 4 Effect on Secondary effect variables.Variables measured are stiffness, patients global assessment of disease severity (PGAD), and quality of life estimated as SF- 12 total, SF- 12 physical (SF FYS) and SF-I 2 Psychological (SF PSYK).Values are given during the course of the study for the active treatment and placebo group patients

\begin{tabular}{|c|c|c|c|c|c|c|c|c|c|c|}
\hline \multirow[b]{2}{*}{ Item } & \multirow[b]{2}{*}{ Week } & \multicolumn{3}{|c|}{ Active } & \multirow[b]{2}{*}{ Wilcoxon } & \multicolumn{3}{|c|}{ Placebo } & \multirow[b]{2}{*}{ Wilcoxon } & \multirow[b]{2}{*}{ Mann-whitney } \\
\hline & & $\mathbf{N}$ & Mean & SD & & $\mathbf{N}$ & Mean & SD & & \\
\hline \multirow[t]{6}{*}{ STIFFNESS } & Week 0 & 60 & 10.48 & 04.30 & & 60 & 09.92 & 04.54 & & 0.4624 \\
\hline & Week 6 & 60 & 09.15 & 04.79 & & 60 & 06.97 & 04.17 & & 0.0116 \\
\hline & DIFF & 60 & 01.33 & 03.99 & 0.0148 & 60 & 02.96 & 04.20 & 0.0001 & 0.0468 \\
\hline & Week 12 & 60 & 08.38 & 04.99 & & 60 & 07.30 & 04.27 & & 0.2974 \\
\hline & DIFF & 60 & 02.10 & 03.69 & 0.0001 & 60 & 02.62 & 04.43 & 0.0001 & 0.3196 \\
\hline & DIFF 6/I2 & 60 & 00.77 & 03.62 & 0.3182 & 60 & 0.34 & 02.45 & 0.7934 & 0.3352 \\
\hline \multirow[t]{6}{*}{ PGAD } & Week 0 & 60 & 05.28 & 02.32 & & 60 & 05.04 & 02.38 & & 0.6124 \\
\hline & Week 6 & 60 & 04.55 & 02.36 & & 60 & 04.22 & 02.50 & & 0.2913 \\
\hline & DIFF. & 60 & 00.74 & 01.88 & 0.0170 & 60 & 00.82 & 02.36 & 0.0168 & 0.8520 \\
\hline & Week 12 & 60 & 04.58 & 02.41 & & 60 & 04.12 & 02.33 & & 0.2202 \\
\hline & DIFF. & 60 & 00.71 & 01.69 & 0.0005 & 60 & 00.92 & 02.13 & 0.0019 & 0.7687 \\
\hline & DIFF 6/I2 & 60 & 0.03 & 01.72 & 0.7572 & 60 & 00.10 & 02.04 & 0.5620 & 0.9958 \\
\hline
\end{tabular}

Citation: Marstrand K,Warholm L, Pedersen F, et al. Dose dependent impact of rose-hip powder in patients suffering from osteoarthritis of the hip and or knee - a double blind, randomized, placebo controlled, parallel group, phase iii study. Int J Complement Alt Med. 20I 7;7(I): I-6. 
Table Continued...

\begin{tabular}{|c|c|c|c|c|c|c|c|c|c|c|}
\hline \multirow[t]{6}{*}{ SF TOTAL } & Week 0 & 57 & 48.34 & 13.68 & & 59 & 50.42 & 12.60 & & 0.3292 \\
\hline & Week 6 & 57 & 51.09 & 14.82 & & 59 & 51.07 & 13.94 & & 0.9493 \\
\hline & DIFF. & 57 & 02.75 & 09.80 & 0.0519 & 59 & 00.65 & 10.29 & 0.6971 & 0.2884 \\
\hline & Week 12 & 57 & 53.18 & $13.6 \mid$ & & 59 & 54.39 & 13.83 & & 0.5232 \\
\hline & DIFF. & 57 & 04.84 & 09.60 & 0.0003 & 59 & 03.96 & 09.69 & 0.0021 & 0.6225 \\
\hline & DIFF $6 / 12$ & 57 & 02.09 & 07.02 & 0.0329 & 59 & 03.32 & 06.85 & 0.0002 & 0.1965 \\
\hline \multirow[t]{6}{*}{ SF FYS } & Week 0 & 57 & 35.50 & 07.86 & & 59 & 37.59 & 06.69 & & 0.0564 \\
\hline & Week 6 & 57 & 37.32 & 07.96 & & 59 & 38.90 & 07.16 & & 0.2244 \\
\hline & DIFF. & 57 & 01.82 & 06.40 & 0.0361 & 59 & 01.31 & 06.17 & $0 .|| 5 \mid$ & 0.5538 \\
\hline & WEEK I 2 & 57 & 38.53 & 07.64 & & 59 & 40.07 & 07.14 & & 0.3966 \\
\hline & DIFF. & 57 & 03.02 & 06.43 & 0.0007 & 59 & 02.48 & 06.50 & 0.0060 & 0.5958 \\
\hline & DIFF $6 / 12$ & 57 & 01.21 & 04.20 & 0.0839 & 59 & 01.17 & 05.53 & 0.1251 & 0.7502 \\
\hline \multirow[t]{6}{*}{ SF PSYK } & Week 0 & 57 & 44.19 & 08.38 & & 59 & 43.56 & 08.7I & & 0.6250 \\
\hline & Week 6 & 57 & 44.27 & 08.94 & & 59 & 42.49 & 09.04 & & 0.2040 \\
\hline & DIFF. & 57 & 00.08 & 05.97 & 0.9659 & 59 & 01.07 & 07.61 & 0.1958 & 0.3274 \\
\hline & Week 12 & 57 & 44.72 & 06.93 & & 59 & 43.91 & 09.74 & & 0.7803 \\
\hline & DIFF. & 57 & 00.53 & 06.46 & $0.907 \mid$ & 59 & 00.35 & 06.76 & 0.8434 & $0.958 I$ \\
\hline & DIFF $6 / 12$ & 57 & 00.45 & 05.70 & 0.4817 & 59 & 01.43 & 05.47 & 0.0230 & 0.2966 \\
\hline
\end{tabular}

In addition, the delta improvement in WOMAC function score was calculated by taking the delta change from start to 12 weeks of treatment. When the delta value was plotted against patients' weight, there was a statistically significant negative correlation coefficient of -0.37 , ( $\mathrm{p}<0.0044)$. The WOMAC function score improved by $21 \%$ in the low weight group $(55-84 \mathrm{~kg})$, whereas the heavier weight group showed no change. A similar calculation of correlation coefficients in the placebo group resulted in a correlation coefficient of -0.01 $(\mathrm{p}<0.938)$. These data support that the actively treated group shows a relationship of ADL improvement to weight of the patients, and indicate a dose-dependency in the treatment group, which was not present in the placebo group at all. A total WOMAC score obtained by pooling WOMAC pain, stiffness and ADL together supported the data given above (Table 3 ).

\section{Secondary effect variables}

Pain and womac function (activity of daily living): Pain and WOMAC function scores were evaluated after 6 weeks. Pain scores in the placebo group were significantly improved as compared to the pain scores observed for the active treatment (Table 2). WOMAC function improved significantly in both the actively treated group and the placebo group with no significant differences between groups (Table 2).

Stiffness: Active treatment resulted in a significant decline in WOMAC symptom score after 6weeks treatment $(\mathrm{p}<0.015)$ and after 12 weeks $(p<0.000)$. Similar results were observed for the placebo group. There was no statistical significant difference comparing the two groups after 12 weeks of treatment $(p<0.320)$ (Table 4). After 6 weeks of treatment the score for the placebo group was slightly better than that observed for active treatment $(\mathrm{p}<0.047)$. On a responder/ non responder basis $62 \%$ responded positively in the active treatment group compared to $70 \%$ in the placebo group. No significant difference comparing groups $(\mathrm{p}<0.440)$.

Dose-dependency: As for pain and WOMAC function the patients with the lower weight, who received active treatment, had the most pronounced reduction in stiffness symptom score. Again, in the placebo group, there was no significant correlation when weight was plotted against WOMAC stiffness symptom score (Table 3).

PGAD: Both placebo and active treatment resulted in a significant reduction in PGAD score after 6 and 12weeks of treatment, with no statistical difference between groups (Table 3). On a responder/ non responder basis $62 \%$ of patients on active treatment responded positively compared to $53 \%$ in the placebo group. There was no statistical difference comparing groups $(\mathrm{p}<0.460)$.
SF-12 Total, SF Physical: Both placebo and active treatment groups showed significant improvements in SF-12 scores, without significant differences between groups (Table 4).

SF-Psychological (mood): Neither active treatment nor placebo resulted in any change in mood (Table 4).

Blood analysis: A minor but significant increase was observed in HDL cholesterol after 12 weeks treatment with Rose-hip $(p<0.036)$. Comparing groups resulted in a p-value of 0.035 in favour of active treatment.

No change was observed in total or LDL-cholesterol (data not given).

The expected seasonal variation in CRP was observed in the placebo group with a $32 \%$ increase (Sung, 2006). In the same time period the active treatment group developed an $18 \%$ reduction $(\mathrm{p}<0.042)$ comparing groups (data not given).

\section{a. Side effects}

No serious side effects were reported. Minor adverse effects, including mild itching and mild gastro intestinal issues, were reported in 6 patients in the actively treated group and by 3 patients in the placebo group.

\section{b. Compliance}

Compliance for the whole 12 week study period was in active treatment: $96.7+/-5.9$; placebo: $98.0+/-3.9$ (P level: 0.222). Thus it was concluded that patients were taking their daily dose of Rose-hip or Placebo.

\section{c. Consumption of medication}

There was no change in the intake of any of the various medications mentioned under characterisation of patients. None of the patients started up new medication during the study period.

\section{d. Dropouts}

Five patients dropped out in the active treatment and 7 in the placebo group.

Dropouts in both groups were reported to be for personal reasons and minor side effects, most often, mild gastro intestinal side effects. No significant difference between groups.

\section{Discussion}

The overall patient group did not show a significant difference between low-dose Rose-hip treatment and placebo in the primary 
outcome measure when evaluating the numeric values (Tables 2 \& 4 ) The percentage of positive responders was approximately $60 \%$ in both groups, with no significant differences between groups.

Analysing the parameters WOMAC pain, function and stiffness for patients of different weight, however, uncovered very interesting findings when using a methodology earlier invented. ${ }^{13}$ This strategy simply focuses, in volunteers all given identical dose of treatment, on a possible correlation between changes in symptom scores and the weight of the volunteer. When this very simple methodology was applied on the present data, a highly significant correlation was found between weight and symptom scores in the active treatment group, for all parts of the WOMAC score system. By contrast, there was no such correlation in the placebo group for any single WOMAC parameter (Table 3). The explanation for this relationship may reflect "dose-dependency" of the effect of Rose-hip. The higher the dose $/ \mathrm{kg}$ bodyweight - the greater the impact on symptoms. This observation is supported by similar findings in another study using $5 \mathrm{~g}$ daily of the same rose hip preparation. ${ }^{13}$ This study was apparently the first to apply a simple "dose-dependency technique" - when testing active treatment vs. placebo in $\mathrm{OA}$.

It is clear that the term "dose-dependency" should be taken with great precaution, and should possibly be changed to "pseudo dose-dependency" as we at present have several factors which we do not control. What is the active ingredient(s) of the rose-hip preparation, how much is absorbed from the gastrointestinal tract of such ingredient(s), what is the plasma concentration and what about hepatic metabolism? However it is interesting, to note, that we in two different studies on the same rose hip preparation have been able to show a relation between the weight of the patient and the outcome of scores on validated questionnaires (WOMAC) and that such relation is not found in groups treated with placebo ${ }^{13}$ for what reason we locally mention this phenomenon: the Winther - Marstrand - Warholm or "WMW pseudo dose-dependency".

The lack of weight dependent effects in the placebo group compared to the finding of weight dependence form the actively treated group, support a specific effect of the Rose-hip treatment in reduction of osteoarthritis symptoms. A high impact from placebo, effect size 0.50 (better than NSAID) was recently reported in a metaanalysis on $\mathrm{OA}$ and is from our experience not surprising at all. ${ }^{16}$

The finding of a symptom score dependency on patient weight provides new insight in studies in which similar clinical effectiveness is observed in active treatment and placebo. An explanation of such findings could be that the correlation reflects a difference in the effective biological concentration achieved when the same dose is given to patients of greatly varying weight as discussed previously. In the current study the weight in each of the two groups in the patient cohort varied from about $60 \mathrm{~kg}$ to $120 \mathrm{~kg}$. One can expect that the low weight patients received the double dose $/ \mathrm{kg}$ body weight, as compared to the heavy weight population - but again we have to take competing factors in consideration.

Although an exact mechanism has not been established for the effect of patient weight and treatment time to develop efficacy, both may reflect the proposal that major and important active ingredients, of which some originate from the seeds of rosehip and some like $\mathrm{GOPO}^{10}$ from the shells, is associated with a lipid soluble component. In fact a lipid soluble main ingredient(s) seems likely, as it takes at least 3 weeks before any symptom reduction is reported and in addition a pronounced "carry over" effect is reported. ${ }^{8}$ Thus the active components distributed in fat stores in heavier individuals, might limit the dose available for the anti-arthritic effects ${ }^{17}$ indicating some precautions for the accuracy of the present WMW methodology.

It is also interesting to note that in a study where the same Rosehip treatment was offered to patients with rheumatoid arthritis, it took 6 months before the number of swollen joints and other markers of symptom reduction had significantly declined. ${ }^{12}$ A similar "pseudo dose relationship" as discussed above, was also defined in that study (personal communication: Kaj Winther).

SF Psychological function (mood) is the only symptom score test, which differs from the pattern described for WOMAC scores and SF physical function. Neither the actively treated nor the placebo treated group changed at all. This result is consistent with the fact that Rosehip was never expected to have any impact on mood, whereas the majority of the population in the Scandinavian countries expected the present product to reduce symptoms of OA.

It is interesting to note that there was a significant difference in favour of active treatment compared to placebo for CRP. CRP significantly increases in the winter season as the result of colds, airway infection and related stresses. ${ }^{18}$ The present study, which ran from late autumn to early winter, showed, as expected for this time of the year that the placebo group developed a significant increase in CRP during the course of the study. It has been shown earlier that CRP can decline as a result of treatment with Rose-hip in higher doses and in the above mentioned study on Rheumatoid arthritis, it was shown that another inflammatory marker SR (sedimentation rate) was significantly lowered after 6 month of Rose-hip treatment, ${ }^{12}$ consistent with the known anti-inflammatory effects of the higher doses of seed and shell containing Rose-hip powder.

The anti-inflammatory mechanism of the present Rose-hip powder supports a greater benefit than its action as a painkiller, and may also explain why it takes a certain time, up to 3 weeks, before symptoms of osteoarthritis starts to decline. ${ }^{8}$ Rose-hip thus appears to work as a disease-modifying agent and not directly as a painkiller, which was also supported by a study indicating improved collagen production as a result of the present rose hip treatment. ${ }^{11}$ Furthermore seeds seam important. ${ }^{19}$ A double blind study testing $40 \mathrm{~g}$ daily of a Rose hip product based on shells only and another similar study testing $2.5 \mathrm{~g}$ daily, were not able to show any impact on inflammatory markers including CRP. ${ }^{20-23}$

Evidence was also obtained that low dose Rose-hip can have some potential cardiovascular protective properties, as HDL cholesterol was significantly elevated.

\section{Conclusion}

Both active treatment and placebo resulted in significant and similar reduction in symptom scores of pain, stiffness and in an improvement of functioning in about $60 \%$ of the patient cohort, so the present design was not able, at first hand, to distinguish any difference between groups, when focusing at the entire groups. However, when the weight of patients was correlated to WOMAC scores it was demonstrated that the effectiveness of the present Rose-hip was greater in the lighter group of patients $(60-84 \mathrm{Kg})$ compared to the heavier group $(85-120 \mathrm{Kg})$, whereas this relations was not found in the placebo group. A likely explanation is that the Rose-hip effectiveness in arthritis symptoms is dose-dependent, the effective dose $/ \mathrm{kg}$ being greater in the lower weight group, as expected by a remedy with true pharmacological activity on osteoarthritis symptoms. This therefore suggests, that the improvement of the approximately $60 \%$ of patients, on active treatment, is based on biochemical actions, different from 
the influence of "expectation" as reported from the placebo group and in many other placebo controlled studies. Overall the current study suggests that patients with osteoarthritis report some benefit after 12 weeks treatment with the low dose Rose-hip regimen, if their weight is less than $84 \mathrm{~kg}$. The present methodology is close to costless as the weight of volunteers and symptom scores are present in most clinical studies testing pain, performance mood and cognitive function. And even though the present "WMW pseudo dose-dependency" should be clearly distinguished from what we normal define as dose-dependency it might be of some interest and use to apply the WMW methodology to other studies where results of active treatment and placebo seams equal at first sight.

\section{Contribution}

All four authors participated in generating the design and protocol. Marstrand K and Warholm L ran the study and collected data and blood samples. All four authors participated in data analysing and in writing the paper.

\section{Role of funding source}

Hyben Vital ApS supplied capsules containing active treatment or placebo and payed technicians for practical work and for analysing and handling of blood samples.

\section{Acknowledgements}

The nurses Kjeldsen $\mathrm{HH}$, Eriksen $\mathrm{S}$, and Tulstrup $\mathrm{B}$ are acknowledged for excellent technical assistance.

\section{Conflicts of interest}

Author declares there are no conflicts of interest.

\section{Funding}

None.

\section{References}

1. Vane JR, Botting RM. Anti-inflammatory drugs and their mechanisms of Action. Inflamm Res. 1998;47(Suppl 2):S78-S87.

2. Griffin MR, Piper JM, Daugherty JR, et al. Nonsteroidal Antiinflammatory Drug Use and Increased Risk for Peptic Ulcerdisease in Elderly Persons. Ann Intern Med. 1991;114(4):257-263.

3. Gislason GH, Jacobsen S, Rasmussen JN, et al. Risk of Death or Reinfarction Associated With the Use of Selective Cyclooxygenase-2 Inhibitors and Nonselective Nonsteroidal Anti-inflammatory Drugs After Acute Myocardial Infarction. Circulation. 2006;113(25):2906-2913.

4. Silverstein FE, Faich G, Goldstein JL, et al. Gastrointestinal Toxicity with Celecoxib vs. Nonsteroidal Anti-inflammatory Drugs for Osteoarthritis and Rheumatoid Arthritis. JAMA. 2000;284(10):1247-1255.

5. Mukherjee D, Nissen SE, Topol EJ. Risk of Cardiovascular events associated with selective Cox-2 inhibitors. $J$ Am Med Assoc. 2001;286(8):954-959.

6. García Rodríguez LA, Hernández-Díaz S. Relative risk of upper gastrointestinal complications among users of acetaminophen and nonsteroidal anti-inflammatory drugs. Epidemiology. 2001;12(5):570-576.
7. Wandel S, Jüni P, Tendal B, et al. Effects of glucosamine, chondroitin, or placebo in patients with osteoarthritis of hip or knee: network metaanalysis. $B M J$. 2010;16:341.

8. Winther K, Apel K, Thamsborg G. A powder made from seeds and shells of a rosehip subspecies (Rosa Canina) reduces symptoms of knee and hip osteoarthritis: a randomized, double-blind, placebo-controlled clinical trial. Scand J Rheumatol. 2005;34(4):302-308.

9. Christensen R, Bartels EM, Altman RD, et al.Does the hip powder of Rosa Canina (Rose-Hip) reduce pain in osteoarthritis patients? A meta-analysis of randomized controlled trials. Osteoarthritis Cartilage. 2008;16(9):965-972.

10. Larsen E, Kharazmi A, Christensen LP, et al. An anti inflamatory galactolipid from Rose-hip (Rosa Canina L.) That inhibits Chemotaxis of Human Peripheral Blood Neutrophils in Vitro. $J$ Nat Prod. 2003;66(7):994-995.

11. Schwager J, Hoeller U, Wolfram S, et al. Rose-hip and its constituent galactolipids confer cartilage protection by modulating cytokine, and chemokine expression. BMC Complement Altern Med. 2011;11:105.

12. Willich SN, Rossnagel K, Roll S, et al. Rose-hip herbal remedy inpatients with rheumatoid arthritis - a randomised controlled trial. Phytomedicine. 2010;17(2):87-93.

13. Winther K, Hansen AS, Kharazmi A. A dose-dependent impact of a standardized powder made from hips of the rose subspecies (Rosa Canina) on WOMAC pain scores in patients with osteoarthritis of the hip and/or knee. Osteoartritis Cartilage. 2009;17(1):253

14. Altman R, Asch E, Bloch D, et al. Development of criteria for the classification and reporting of osteoarthritis. Classification of osteoarthritis of the knee. Arthritis Rheum. 1986;29(8):1039-1049.

15. Altman R, Alarcon G, Appelrouth D, et al. The American College of Rheumatology criteria for the classification and reporting of osteoarthritis of the hip. Artthritis Rheum. 1991;34(5):505-514.

16. Zhang W, Robertson J, Jones AC, et al.The placebo effect and its determinants in osteoarthritis: meta-analysis of randomised controlled trials. Ann Rheum Dis. 2008;67(12):1716-1723.

17. Christensen R, Bartels EM, Riecke BF, et al. Improved nutritional status and bone health after diet-induced weight loss in sedentary osteoarthritis patients: a prospective cohort study. Eur J Clin Nutr.2012;66(4):504-509.

18. Sung KC. Seasonal variation of $\mathrm{C}$ reactive protein in apparently healthy Koreans. Int J Cardiol. 2006;107(3):338-342.

19. Winther K, Hansen ASV, Campbell-Tofte J. Bioactive ingredients of rose hips (Rosa caninaL) with special reference to antioxidative and anti-inflammatory properties: in vitro studies. Botanics: Targets and Therapy. 2006;6:11-23.

20. Andersson U, Berger K, Högberg A, et al. Effects of rose hip intake on risk markers of type 2 diabetes and cardiovascular disease: a randomized, double-blind, cross-over investigation in obese persons. Eur J Clin Nutr. 2012;66(5):585-590.

21. Ginnerup-Nielsen E, Christensen R, Bliddal H, et al. Improved gait in persons with knee relaterd mobility limitations by a rosehip food supplement: A randomized, double-blind, placebo-controlled trial. Gait and Posture. 2015;42(3):340-347.

22. Marstrand $\mathrm{K}$ and Campbell-Tofte J. The role of rose hip (Rosa canina L) powder in alleviating arthritis pain and inflammation - part II animal and human studies. Botanics: Targets and Therapy. 2016;6:59-73. 